\title{
Radio Networks Predictive Control of a T-type Inverter in Wind Energy
}

\author{
https://doi.org/10.3991/ijoe.v12i12.6456 \\ Jian-jun Zhu ${ }^{1}$,Guo-liang Yang ${ }^{2}$, Yang $\mathrm{Zi}^{2}$, Yi-ming Fang ${ }^{2}$ \\ ${ }^{1}$ Jilin Institute of Chemical Technology, JiLin,China \\ ${ }^{2}$ Yan Shan University, Qinhuangdao, China
}

\begin{abstract}
A new controlling method of three level inverters is proposed in this paper. Three-level $T$ inverters are widely used in industry for high-power, medium-voltage power conversion and drives. Topics related to power losses due to commutation and quality of the output current is relevant issues in this power range. The neutral-point balancing problem in this topology is another subject that has been studied in recent years. Among the most common control methods for this converter, the literature states are non linear techniques, like hysteresis control, and linear methods, like the use of PI controllers in conjunction with pulse width modulation. The general predictive control scheme is applied here to the inverter. The behavior of the system is predicted for each possible switching state of this kind of inverter. The switching state that minimizes a given cost function is selected to be applied during the next sampling interval following the same strategy.
\end{abstract}

Index Terms-Predictive Control; T-type Inverter; Grid Connected

\section{INTRODUCTION}

Recently, the three-level T-type inverter has been proposed for high-efficiency systems in low-voltage applications such as photovoltaic (PV) inverter, power factor corrector (PFC) rectifier, and automotive inverter systems ${ }^{[1]}$. Fig. 1.shows the simplified circuit of a T-type inverter.

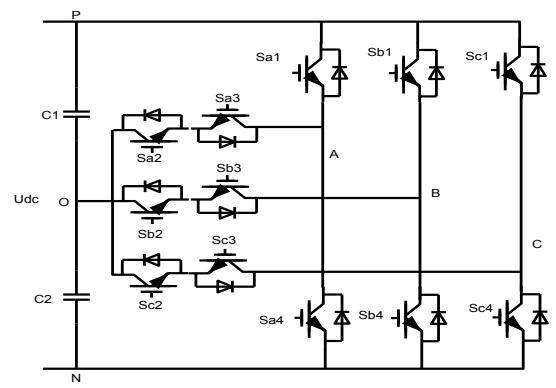

Figure 1. T-type inverter

The T-type inverter uses the same switch as that used in a conventional two level inverter because these switches ( $S_{x 1}(x=a, b, c)$ and $\left.S_{x 4}\right)$ have to block the full dc-link voltage. A bidirectional switch is connected between the neutral point and each output. Unlike the half-bridge switches $\left(S_{x 1}\right.$ and $\left.S_{x 4}\right)$, the bidirectional $\operatorname{es}\left(S_{x 2}\right.$ and $\left.S_{x 3}\right)$ have to block only half of the dc-link voltage. Therefore, it is possible to use the devices having a lower voltage rating. The neutral-point clamped (NPC) inverter uses the two switches connected in series to block the full dc-link voltage. On the other hand, the T-type inverter uses a single switch to block the full dc-link voltage. Therefore, the conduction losses of the T-type inverter are considerably reduced compared to that of the NPC inverter. The T-type inverter has reduced switching losses and switching noise because the neutral-point switches are operated under the half of the dc-link voltage. Therefore, the total losses in a T-type inverter are the lowest among those of the two-level, NPC, and T-type inverters in medium switching frequencies $(4-30 \mathrm{kHz})$.

This configuration allows the generation of three voltage levels at the output terminal of phase, with respect to the neutral point 0 , considering the switching combinations given in Table 1 .

TABLE I.

SWITCHING STATES FOR ONE PHASE OF THE INVERTER

\begin{tabular}{|c|c|c|c|c|c|}
\hline$S_{x}$ & $S_{x 1}$ & $S_{x 2}$ & $S_{x 3}$ & $S_{x 4}$ & $v_{x o}$ \\
\hline+ & 1 & 1 & 0 & 0 & $V_{\mathrm{dc}} / 2$ \\
\hline 0 & 0 & 1 & 1 & 0 & 0 \\
\hline- & 0 & 0 & 1 & 1 & $-V_{\mathrm{dc}} / 2$ \\
\hline
\end{tabular}

For the three phases of the inverter, 27 switching states are generated, which produce 19 different voltage vectors, as shown in Fig.2. Note that some switching states are redundant, generating the same voltage vector. The three levels $\mathrm{T}$ inverter presents a high number of switching states, compared to the two-level inverter. The larger set of possible actuations allows for additional degrees of freedom and several compositions of the cost function can be considered ${ }^{[2]}$.

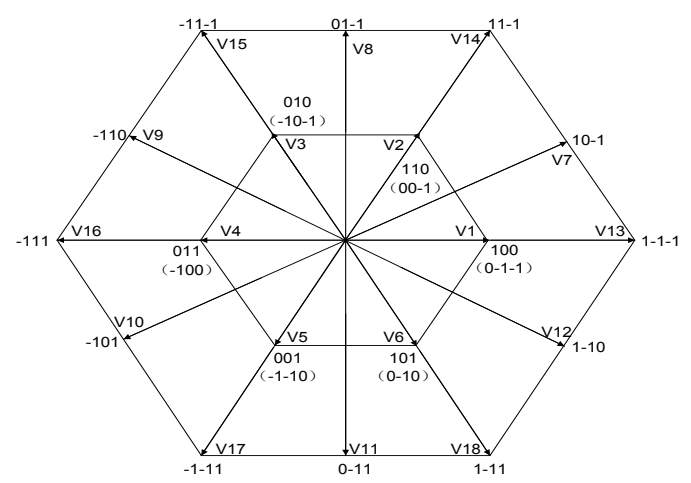

Figure 2. Possible voltage vectors and switching states generated by a three-level inverter 
The general predictive control scheme is applied here to the T-type inverter ${ }^{[3]}$. The behaviour of the system is predicted for each possible switching state of this kind of inverter. The switching state that minimizes a given cost function is selected to be applied during the next sampling interval. The T-type inverter presents a high number of switching states, compared to the two-level inverter. The larger setoff possible actuations allow for additional degrees of freedom and several compositions of the cost function can be considered. Considering the control requirements that are characteristic of this topology and its applications, several variations of the algorithm are studied and compared to classical linear control with PWM, including features like load current reference tracking, balance in the DC link capacitor voltages, and reduction of the switching frequency ${ }^{[4]}$.

With the advancements in the microprocessor technology ${ }^{[5]}$, the predictive control has found many applications in the electrical power conversion systems. The finite control-set model predictive control (FCS-MPC) is a class of controllers that are most suitable for the power electronic converters. This controller is intuitive, easy to implement and it can be applied to variety of systems with easy inclusion of constraints and/or nonlinearities.

In this paper, this control strategy can be summarized in the following steps:

1. Define a cost function $\mathrm{g}$.

2. Build a model of the converter and its possible switching states.

3. Build a model of the Grid connected for prediction.

A discrete-time model of the load is needed to predict the behaviour of the variables evaluated by the cost function, that is, the grid currents.

\section{Predictive Current Control Strategy}

\section{A. System Model}

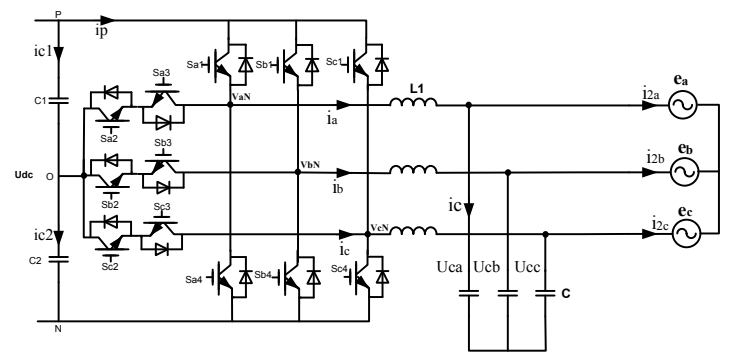

Figure 3. Grid connected model of T-type inverter

\section{1) Grid Model}

Taking into account the definitions of variables from the circuit shown in Figure3, leaving out the currents flow by the capacitors, the equations for load current dynamics for each phase can be written as

$$
\begin{gathered}
v_{a N}=L \frac{d i_{a}}{d t}+R i_{a}+e_{a} \\
v_{b N}=L \frac{d i_{b}}{d t}+R i_{b}+e_{b} \\
v_{c N}=L \frac{d i_{c}}{d t}+R i_{c}+e_{c}
\end{gathered}
$$

Where $\mathrm{R}$ is the load resistance and $\mathrm{L}$ the load inductance.

Considering the unitary vector $a=e^{j 2 \pi / 3}$, which represents the $120^{\circ}$ phase displacement between the phases, the output voltage vector can be defined as

$$
v=\frac{2}{3}\left(v_{a N}+a v_{b N}+a^{2} v_{c N}\right)
$$

\section{2) Discrete-Time Model for Prediction}

The discrete-time model will be used to predict the future value of grid current from voltages and measured currents at the kith sampling instant ${ }^{[6]}$. Several discretization methods can be used in order to obtain a discrete-time model suitable for the calculation of predictions. Considering that the grid can be modeled as a first-order system, the discrete-time model can be obtained by a simple approximation of the derivative. However, for more complex systems this approximation may introduce errors into the model and a more accurate discretization method is required.

The grid current derivative $d i / d t$ is replaced by a forward Euler approximation. That is, the derivative is approximated as follows:

$$
\frac{d i}{d t} \approx \frac{i(k+1)-i(k)}{T_{s}}
$$

which is substituted in (5) to obtain an expression that allows prediction of the future load current at time $k+1$, for each one of the 27 values of voltage vector $v(k)$ generated by the inverter. This expression is

$$
i^{p}(k+1)=\left(1-\frac{R T_{S}}{L}\right) i(k)+\frac{T_{s}}{L}(v(k)-\hat{e}(k))(6)
$$

Where $\hat{e}(k)$ denotes the estimated back-emf. The superscript $\mathrm{p}$ denotes the predicted variables.

The reference currents can be calculated from considering the power reference $P^{*}=1 \mathrm{kw}, Q^{*}=0$. According to the theory of instantaneous power ${ }^{[7]}$, The instantaneous active and reactive power of the system can be calculated with the following expression;

$$
\left[\begin{array}{l}
P \\
Q
\end{array}\right]=\frac{3}{2}\left[\begin{array}{cc}
e_{d} & e_{q} \\
e_{q} & -e_{d}
\end{array}\right]\left[\begin{array}{l}
i_{d} \\
i_{q}
\end{array}\right]
$$

So it's obvious to get the reference currents

$$
\left.\left[\begin{array}{l}
i_{d}^{*} \\
i_{q}^{*}
\end{array}\right]=\frac{2}{3\left[\left(e_{d}\right)^{2}+\left(e_{q}\right)^{2}\right.}\right]\left[\begin{array}{cc}
e_{q} & -e_{d} \\
e_{d} & e_{q}
\end{array}\right]\left[\begin{array}{l}
p^{*} \\
q^{*}
\end{array}\right]
$$

The back-emf can be calculated from (6) considering measurements of the load voltage and current with the following expression;

$$
\hat{e}(k-1)=v(k-1)-\frac{L}{T_{s}} i(k)-\left(R-\frac{L}{T_{s}}\right) i(k-1)^{(9)}
$$$$
\wedge
$$

Where $e(k-1)$ is the estimated value of $e(k-1)$. The present back-emf $e(k)$, can be estimated using an extrapolation of the past values of the estimated back-emf. 
Alternatively, as the frequency of the back-emf is much less than the sampling frequency, we will suppose that it does not change considerably in one sampling interval and, thus, assume $\hat{e}(k)=\hat{e}(k-1)$. The same approximation of the derivative can be used for the capacitor voltages for a sampling time $T_{s}$

$$
\frac{d v_{c x}}{d t} \approx \frac{v_{c x}(k+1)-v_{c x}(k)}{T_{s}}
$$

giving the following discrete-time equations:

$$
\begin{aligned}
& v_{c 1}^{p}(k+1)=v_{c 1}(k)+\frac{1}{c} i_{c 1}(k) T_{s} \\
& v_{c 2}^{p}(k+1)=v_{c 2}(k)+\frac{1}{c} i_{c 2}(k) T_{s}
\end{aligned}
$$

where currents $i_{c 1}(k)$ and $i_{c 2}(k)$ depend on the switching state of the inverter and the value of the output currents, and can be calculated using the following expressions:

$$
\begin{gathered}
i_{c 1}(k)=i_{d c}(k)-H_{1 a} i_{a}(k)-H_{1 b} i_{b}(k)-H_{1 c} i_{c}(k) \\
i_{c 2}(k)=i_{d c}(k)+H_{2 a} i_{a}(k)+H_{2 b} i_{b}(k)+H_{2 c} i_{c}(k)
\end{gathered}
$$

Where $i_{d c}$ is the current supplied by the voltage source $V_{d c}$. Variables $H_{1 x}$ and $H_{2 x}$ depend on the switching states and are defined as

$$
\begin{aligned}
& H_{1 x}=\left\{\begin{array}{l}
1, \text { if } S_{x}="+" \\
0, \text { otherwise }
\end{array}\right. \\
& H_{2 x}= \begin{cases}1, & \text { if } S_{x}="-" \\
0, \text { otherwise }\end{cases}
\end{aligned}
$$

with $x=a, b, c$.

Hence, (9)-(12) allow us to predict the effect of selecting a given switching state on the variation of the capacitor voltages ${ }^{[8]}$.

\section{B. Predictive Current Control Method}

The control requirements for the T-type inverter are:

1. Grid current reference tracking

2. DC link capacitor voltages balance

3. Reduction of the switching frequency

4. These requirements can be formulated in the form of a cost function to be minimized. The cost function for the T-type inverter has the following composition (equation 17):

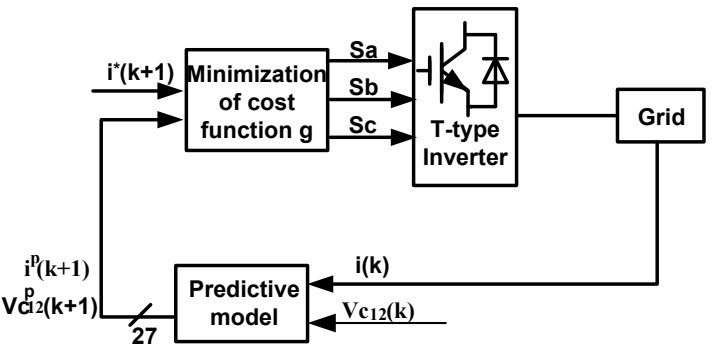

Figure 4. Predictive current control method for the T-type inverter

$g=\left|i_{\alpha}^{*}-i_{\alpha}^{p}\right|+\left|i_{\beta}^{*}-i_{\beta}^{p}\right|+\lambda_{d c}\left|v_{c 1}^{p}-v_{c 2}^{p}\right|+\lambda_{n} n_{c}$

The first two terms are the load current errors in orthogonal coordinates, where $i_{\alpha}^{p}$ and $i_{\beta}^{p}$ are the real and imaginary components of the predicted current vector $i^{p}$, respectively, and $i_{\alpha}^{*}$ and $i_{\beta}^{*}$ are the real and imaginary components of the reference current vector $i^{*}$.

The third term in the cost function measures the difference in the predicted values of the DC link capacitor voltages. These predicted voltages are calculated using (11) and(12). Then, by minimization of this term, the capacitor voltages will tend to be equal.

The last term is the number of commutations required to switch from the present switching state to the switching state under evaluation. A switching state that implies fewer commutations of the power semiconductors will be preferred. In this manner, the use of this term will have a direct effect on the switching frequency of the converter $[9]$.

Weighting factors $\lambda_{d c}$ and $\lambda_{n}$ handle the relation between terms dedicated to reference tracking, voltage balance, and reduction of switching frequency within the cost function. A large value of certain $\lambda$ implies greater priority to that objective.

\section{COST FunCtiON SELECTION}

\section{A. Minimization of the Switch Frequency}

NAs in power converters, one of the major measures of control effort are the switching frequency. It is important in many applications to be able to control or limit the number of commutations of the power switches ${ }^{[10]}$.

To directly consider the reduction in the number of commutations in the cost function, a simple approach is to include a term in it that covers the number of switches that change when the switching state $S(k)$ is applied, with respect to the previously applied switching state $S(k-1)$.

The resulting cost function is expressed as $g=\left(i_{\alpha}^{*}-i_{\alpha}^{p}\right)+\left(i_{\beta}^{*}-i_{\beta}^{p}\right)+\lambda_{n} \cdot n \quad$.Considering the three-phase inverter as an example, the switching state vector $S=\left(S_{a}, S_{b}, S_{c}\right)$, defines the switching state of 
each inverter leg ${ }^{[11]}$. Then the number of switches changing from time $k-1$ to time $k$ is

$$
n=\left|S_{a}(k)-S_{a}(k-1)\right|+\left|S_{b}(k)-S_{b}(k-1)\right|+\left|S_{c}(k)-S_{c}(k-1)\right|
$$

\section{B. Capacitor Voltage Balance}

One of the most interesting aspects of the predictive control method is the simplicity for implementing voltage balance in the DC link ${ }^{[12]}$. This feature was tested by disconnecting the middle point of the DC link from the source and applying the predictive control method with the following cost function:

$$
g=\left|i_{\alpha}^{*}-i_{\alpha}^{p}\right|+\left|i_{\beta}^{*}-i_{\beta}^{p}\right|+\lambda_{d c}\left|v_{c 1}^{p}-v_{c 2}^{p}\right|
$$

Nevertheless, to take advantage of the possibilities offered by this control method, it is necessary to adjust parameters $\lambda_{d c}$ and $\lambda_{n}$. Some design procedures should be established for this purpose. First, the designer should consider the different units and magnitudes of the variables involved in the cost function $\mathrm{g}^{[13]}$. This will give some idea about the order of magnitude of the weighting factors for equal importance of all terms. If the designer wants to maintain voltage balance in the DC link only by selecting the appropriate switching state from the redundant states that generate a given voltage vector, then a small value of $\lambda_{d c}$ should be used. The smallest value allowed by the implementation platform will work for that purpose. The same criteria can be applied to $\lambda_{n}$. With a small value; the method will choose the switching state that implies fewer commutations within a voltage vector. When increasing $\lambda_{n}$, the method could choose switching states that are not within the optimal voltage vector in terms of reference tracking, but imply fewer commutations.

Summarizing, the predictive current control method was implemented, confirming the observations made in simulations. The strategy succeeded in maintaining voltage balance in the DC link and reducing the switching frequency. Working at the same switching frequency, the presented method achieved better reference tracking than the carrier-based method.

\section{Simulation}

The predictive current control strategy is implemented for simulation in MATLAB asaS-Function, containing the code in Figure 5.

When the predictive control is implemented experimentally, the same code is rewritten in C language with alpha and beta currents calculated separately ${ }^{[14]}$. Results using the control algorithm implemented in MATLAB/Simulink are shown next, considering (6) for load current prediction and (9) for back-emf estimation. The system parameters $\mathrm{V}_{\mathrm{dc}}=800 \mathrm{v}, \mathrm{L}=10 \mathrm{mH}, \mathrm{R}=0.02 \Omega$, and $\mathrm{e}=311 \mathrm{~V}_{\text {peak }}$ have been considered for simulations. Current and voltage in one phase of the load are shown in Fig.6 for a sampling time $T_{s}=10 \mu F$. There is no steady state error in the current but there is a noticeable ripple. This ripple is reduced considerably when a smaller sampling time is used at sampling time $T_{s}=3 \mu F$. However, by reducing the sampling time, the switching frequency is increased as can be seen by comparing the load voltages in Fig.7.

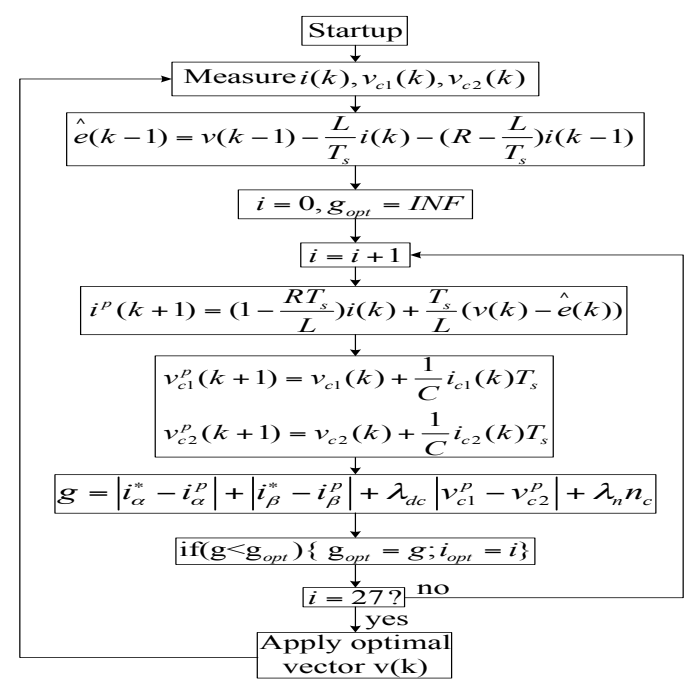

Figure 5. Flow diagram of the implemented control algorithm
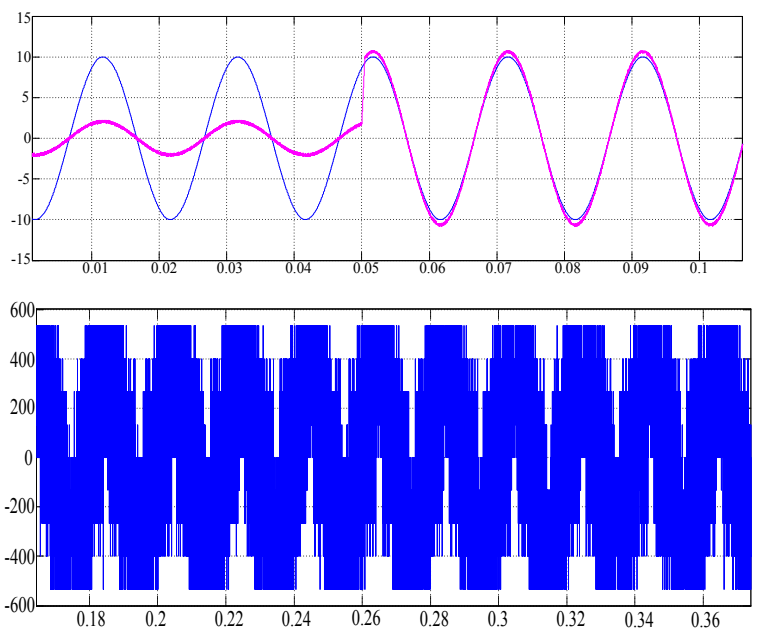

Figure 6. Phase voltage and phase current output voltage of phase A
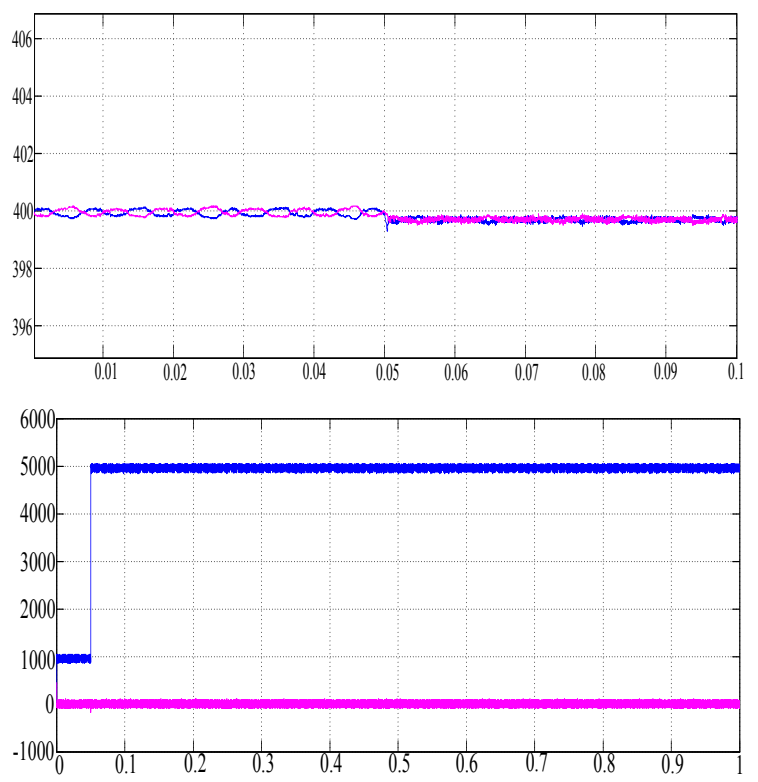

Figure 7. Capacitor voltage of $\mathrm{C} 1, \mathrm{C} 2$ power tracking 
However, the proposed method requires a greater sampling frequency or data acquisition frequency. The previous fact should not be a problem, considering the new technologies available for DSPs. It is important to mention that the sampling instant is always located at a fixed position within the sampling period, making easy the acquisition of measurement data, and avoiding problems with switching the power devices [15]. The dSPACE system used to obtain the results had no problem running the algorithm at the sampling time selected $\mathrm{Ts}=100 \mu \mathrm{s}$, In fact, it took only $52 \mu \mathrm{s}$ to execute the entire algorithm, including voltage balance and reduction of the switching frequency. The algorithm was also implemented on a DSP from Texas Instruments, TMS320F2812, using the same sampling frequency and achieving similar results in terms of processing times.

\section{CONCLUSIONS}

The predictive current control method presented does not require any kind of linear controller or modulation technique. It effectively controls the load current and compares well to established control methods like PWM, achieves a comparable dynamic response and reference tracking, and works at lower switching frequencies. One of the remarkable aspects of the method is the use of costs assigned to each objective to achieve reference tracking, balance in the DC link, and a reduction in the switching frequency. The simplicity of the theory makes it easy to understand and implement. The strategy allows the designer to adjust the $\lambda$ parameters to fit the requirements in terms of switching frequency, voltage balance, and reference tracking. A systematic way to determine the weighting factors is a challenge for future work.

\section{REFERENCES}

[1] A. Nabae, I. Takahashi, and H. Akagi, "A new neutral-pointclamped PWM inverter," IEEE Transactions on Industry Applications, vol. IA-17, no. 5, pp. 518 - 523, September/October 1981. https://doi.org/10.1109/TIA.1981.4503992

[2] B. Wu, "High-power converters and AC motor drives," Power Electronics Specialists Conference, PESC' 05, 2005.

[3] Jana K C, Biswas S K. Generalised switching scheme for a space vector pulse-width modulation-based $\mathrm{N}$-level inverter with reduced switching frequency and harmonics[J]. Iet Power Electronics, 2015, 8(12):2377-2385. https://doi.org/10.1049/ietpel. 2015.0101

[4] Latran M B, Teke A. Investigation of multilevel multifunctional grid connected inverter topologies and control strategies used in photovoltaic systems[J]. Renewable \& Sustainable Energy Reviews, 2015, 42(42): 361 - 376. https://doi.org/10.1016/ j.rser.2014.10.030
[5] Cui B. T-type three-level inverter circuit: US, US8665619[P]. 2014.

[6] R. Vargas, P. Cortés, U. Ammann, J. Rodríguez, and J. Pontt, "Predictive control of a three-phase neutralpoint-clamped inverter," IEEE Transactions on Industrial Electronics, vol. 54, no. 5, pp. 2697 - 2705, October 2007. https://doi.org/10.1109/TIE.2007. $\underline{899854}$

[7] Cortes P, Rodriguez J, Silva C, et al. Delay Compensation in Model Predictive Current Control of a Three-Phase Inverter[J]. IEEE Transactions on Industrial Electronics, 2012, 59(2):13231325. https://doi.org/10.1109/TIE.2011.2157284

[8] J. Holtz, "Pulse width modulation for electronic power conversion,” Proceedings of the IEEE, vol. 82, no. 8, pp. $1194-1214$, August 1994. https://doi.org/10.1109/5.301684

[9] M.J. Duran, J. Prieto, F. Barrero, and S. Toral, "Predictive current control of dual three-phase drives using restrained search techniques,” IEEE Transactions on Industrial Electronics, vol. 58, no. 8 , pp. 3253 - 3263,August 2011. https://doi.org/10.1109/ TIE.2010.2087297

[10] R.Vargas,J. Rodríguez, U. Ammann, and P. W. Wheeler, “Predictive current control of an induction machine fed by a matrix converter with reactive power control," IEEE Transactions on Industrial Electronics, vol. 55, no. 12, pp. 4362 - 4371, December 2008. https://doi.org/10.1109/TIE.2008.2006947

[11] P. Cort' es, J. Rodríguez, D. E. Quevedo, and C. Silva, "Predictive current control strategy with imposed load current spectrum," IEEE Transactions on Power Electronics, vol. 23, no. 2, pp. 612 - 618, March2008. https://doi.org/10.1109/ TPEL.2007.915605

[12] Rodriguez J, Cortes P. Predictive Control of Power Converters and Electrical Drives[M]. West Sussex, John Wiley \& Sons Ltd, 2012 https://doi.org/10.1002/9781119941446

[13] Xia Changliang, Liu Tao, Shi Tingna, et al. A Simplified Finite Control Set Model Predictive Control for Power Converters[J]. IEEE Transactions on Industrial Informatics. 2013,28(99):1-12

[14] Cortes P,Wilson A, Kouro S, et al. Model Predictive Control of Multilevel Cascaded H-Bridge Inverters[J]. IEEE Transactions on Industrial Electronics, 2010,57(8):2691-2699 https://doi.org/10.1109/TIE.2010.2041733

[15] Rodriguez J, Cortes P. Predictive control of power converters and electrical drives[J]. Predictive Control of Power Converters \& Electrical Drives, 2012, 6(4):1785 - 1786 https://doi.org/10.1002/9781119941446

\section{AUTHORS}

Jian-jun Zhu "Control study of motor process and intelligent”, M.D., Dept. Automaton., Jilin Institute of Chemical Technology, Ji Lin,China, (zjj099@163.com).

Guo-liang Yang "Control study of motor new energy power generation, power electronics and intelligent", M.D., Dept. Electrical Engineering and Automation, Yan Shan University, Qinhuangdao, China, 1996.

Submitted 26 October 2016. Published as resubmitted by the authors 27 November 2016. 